\title{
What variety of attention is automatically captured by peripheral cues?
}

\author{
STEVEN J. LUCK and STEPHEN J. THOMAS \\ University of Iowa, Iowa City, Iowa
}

\begin{abstract}
Previous studies have shown that the voluntary allocation of attention to a location in space can influence accuracy in two ways. First, additional processing resources can be allocated to the attended location, leading to an improvement in perceptual quality for objects presented at that location. Second, decision processes can be restricted to information arising from the attended location, which improves accuracy without influencing the perceptual representation. The present study examined the operation of these two attentional mechanisms when nonpredictive peripheral cues were used to capture attention automatically. Experiment 1 showed that, like predictive cues, nonpredictive cues influence accuracy by summoning perceptual resources and also by influencing decision processes. However, both the cues and the targets in this experiment were defined by luminance increments, making it possible that the cuing effects were mediated by a task-controlled attentional set rather than being fully automatic. Experiments 2 and 3 examined this possibility by using luminance-defined cues and color-defined targets; evidence was again obtained for both perceptual-level and decision-level attention effects. The capture of attention by a nonpredictive peripheral cue thus appears to influence both perceptual resource allocation and postperceptual decision processes.
\end{abstract}

Research on selective attention has typically focused on two fundamental issues, the control of attention and the effects of attention. Studies of attentional control examine the principles that determine which sources of information are attended at a given moment, and studies of the effects of attention examine differences in information processing that occur as a consequence of the allocation of attention. The present study addresses the intersection of these two issues by examining the nature of the attention effects produced by a specific type of attentional control mechanism. Specifically, this study examines whether the automatic capture of attention produced by nonpredictive peripheral cues reflects the allocation of perceptual processing resources to the cued location, as is commonly assumed, or whether automatic attention effects can be explained by changes in decision processes that yield more accurate discrimination performance without influencing the quality of the perceptual representation. Previous studies have shown that both of these types of attention effects may be present when attention is directed voluntarily (Henderson, 1996; Lappin \& Uttal, 1976; Luck, Hillyard, Mouloua, \& Hawkins, 1996; Shiu

S.J.T. is now at EEG Systems Laboratories, San Francisco. This study was supported by Grant 95-38 from the McDonnell-Pew Program in Cognitive Neuroscience and by a Grant-in-Aid of Research from the National Academy of Sciences, through Sigma Xi, the Scientific Research Society. The authors would like to thank Steve Yantis and Jim Johnston for their excellent comments and suggestions on a previous version of the manuscript. Correspondence should be addressed to $S$. J. Luck, Department of Psychology, University of Iowa, 11 Seashore Hall E, lowa City, IA 52242-1407 (e-mail: steven-luck@uiowa.edu).
\& Pashler, 1994), but this issue has never been explicitly examined in the case of exogenous attentional control.

We will begin our presentation of this issue by describing previous studies of resource allocation and decision effects, and we will then discuss how these effects might be influenced by endogenous and exogenous attentional control mechanisms.

\section{Resource Allocation and Uncertainty Reduction Models}

Across a wide variety of contexts, stimuli presented within the focus of attention are typically found to be processed more quickly and accurately than stimuli presented outside the focus of attention. This has been studied most commonly with the spatial cuing paradigm, in which a cue stimulus is used to direct attention to a particular location and a target stimulus is then presented at either the cued location or an uncued location. Target discrimination is typically found to be faster or more accurate when the target appears at the cued location (i.e., on valid trials) than when the target appears at an uncued location (i.e., on invalid trials), and effects of this nature have been found across a wide variety of experimental conditions (see, e.g., Cheal \& Lyon, 1991; Eriksen \& Hoffman, 1974; Hawkins, Shafto, \& Richardson, 1988; Jonides, 1981; Reinitz, 1990). These effects are usually explained in terms of resource allocation models, which propose that attention is used to distribute a limited pool of perceptual processing resources among multiple locations for the purpose of maximizing overall performance (see, e.g., Kahneman, 1973). In most cuing experiments, the targets usually appear at the cued location and only rarely appear at an uncued location, and the allocation of 


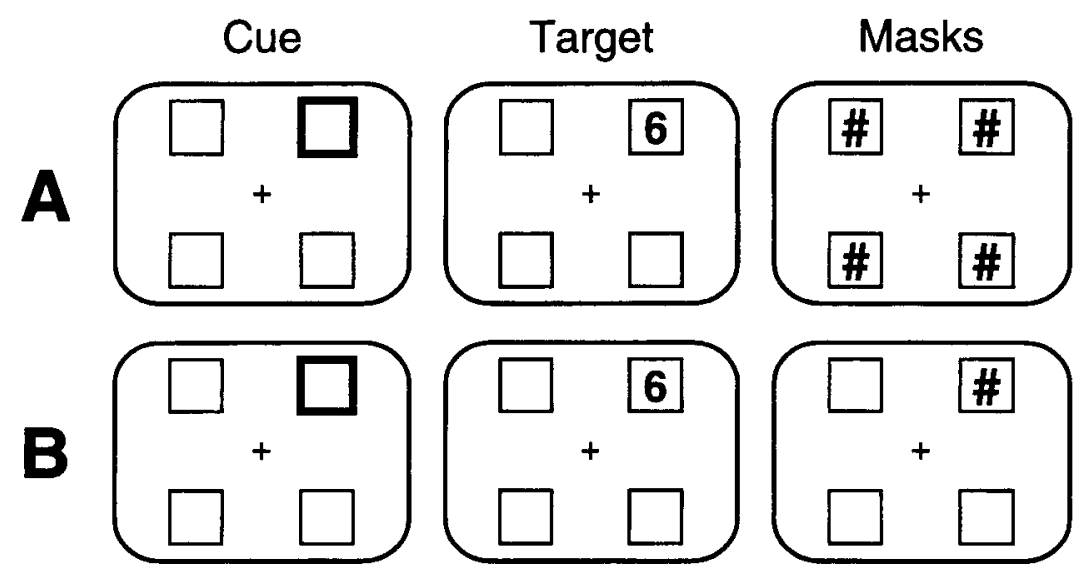

Figure 1. Example of a cuing experiment in which the location of the target is difficult to perceive because of the presence of multiple masks $(A)$ or easy to perceive because of the presence of a single mask (B).

resources to the cued location is therefore useful for maximizing performance. When peripheral luminance transients are used as cues, however, attention may be allocated to the cued location even if the target is unlikely to appear at that location. This would not maximize performance in an artificial experimental context, but it may be useful in the natural environment, where luminance transients often signal important events such as the appearance of a new object (Egeth \& Yantis, 1997).

Although resource allocation models are commonly used to explain cuing effects, several investigators have proposed an alternative account that is based on decision factors rather than changes in perceptual processing (see, e.g., Eriksen \& Hoffman, 1974; Lappin \& Uttal, 1976; Shaw, 1984; Sperling \& Dosher, 1986). A particularly forceful description of this model was provided by Shiu and Pashler (1994), who argued that the discrimination of alphanumeric characters does not require access to any limited-capacity resources and that the effects of attention on accuracy for such stimuli can be explained by decision factors that would be present even in an ideal observer with unlimited capacity. As an example of their argument, consider the cuing experiment illustrated in Figure 1A, in which each trial consists of a sequence of three events, an arrow that points to one of four locations as a cue, followed by a digit at one of the four locations that serves as a target, and finally a set of \# symbols at all four locations that serve as masks that bring accuracy away from ceiling. In such an experiment, the presence of masks at all four locations may make it difficult to be certain which location contained the target stimulus; consequently, the observer's discrimination may be influenced by noise arising from all four locations in addition to the signal at the location of the target. For example, the observer may have a faint perception of a 6 at the location of the target due to the presence of the target at that location, but he/she may also have a faint perception of a 4 at another location due to a combination of neural noise, environmental noise, and the shape of the masking stimulus. To perform the discrimination task, the observer must decide which of the two faint percepts should be reported, and this decision is obviously very important in determining whether the observer's response will be correct or incorrect.

According to the argument of Shiu and Pashler (1994), cues operate solely by influencing which of the percepts will be reported. Specifically, if the cue stimulus correctly indicates the location of the subsequent target stimulus on the majority of trials, then the observer can maintain a higher level of accuracy by always reporting the percept arising from the cued location and disregarding percepts arising from the uncued locations. ${ }^{1}$ This will lead to much greater accuracy on valid trials, because the observer will report the percept arising from the location of the actual target and will disregard noise-induced percepts arising from the other locations. However, this will lead to very inaccurate performance on invalid trials, because the observers will be reporting a noise-based percept arising from the cued location rather than the percept arising from the actual target. We refer to this as the uncertainty reduction model to denote the proposed role of the cue stimulus in reducing the observer's uncertainty about which location contains the target.

It is important to note that the uncertainty reduction model posits that the quality of the target representation is not changed in any way by attention: Attention merely influences the way in which the observer uses the representations to make a response. This contrasts with resource allocation models, which propose that the quality of the target representation is improved when additional resources are available for processing the target.

\section{Testing the Resource Allocation and Uncertainty Reduction Models}

Shiu and Pashler (1994) tested the uncertainty reduction model by determining whether attention effects would be eliminated if observers could easily determine 
which location contained the target stimulus. This was accomplished by comparing a condition in which the location of the target was obscured by the presence of masks at all four locations, as in Figure 1 A, with a condition in which the location of the target was made obvious by presenting a mask only at the target location, as in Figure 1B. If cues operate solely by influencing which location is reported and do not lead to the allocation of processing resources to the cued location, then the cues should be ineffective when the location of the target is obvious. In other words, if the target is followed by a single mask at the same location, there is no uncertainty regarding the target location, and observers will report the percept arising from the target location whether it was cued or not. Shiu and Pashler predicted that discrimination accuracy would be equal on valid and invalid trials in the singlemask condition, in which the location of the target was obvious. In accord with this prediction, they found greater accuracy on valid trials than on invalid trials in the multiple-mask condition, but no effects of cue validity in the single-mask condition. They also reviewed a large number of previous studies in which accuracy was measured as a function of cue validity, and they concluded that significant cue validity effects were observed only in experiments in which the location of the target was not obvious. Many studies have shown significant validity effects on reaction time in the absence of location uncertainty, but Shiu and Pashler attributed these results to speed-accuracy tradeoffs. ${ }^{2}$ In sum, they concluded that attention does not lead to improved discrimination of alphanumeric characters by increasing the availability of perceptual processing resources, but instead operates by influencing decision processes.

Although Shiu and Pashler (1994) provided strong evidence that cue validity effects can be caused by a modulation of decision processes, subsequent studies demonstrated that such effects can also arise due to changes in resource allocation (Cheal \& Gregory, 1997; Henderson, 1996; Luck et al., 1996). For example, Luck et al. (1996) demonstrated that significant cue validity effects could be obtained under a variety of conditions with a single target and mask and, hence, no uncertainty about target location (see also Hawkins et al., 1990; Luck et al., 1994). Thus, the voluntary allocation of attention to a location in space leads to changes in both perceptual-level resources and decision-level processes.

\section{Exogenous Versus}

\section{Endogenous Attentional Control}

A great many studies have examined the automatic orienting of attention produced by peripheral luminance transients (e.g., Jonides, 1981; Posner \& Cohen, 1984; Yantis \& Jonides, 1990). However, none of these studies has addressed the issue of whether automatic attentional orienting leads to changes in perceptual resource allocation or to changes in postperceptual decision processes. In fact, almost all studies of purely automatic orienting have focused on reaction time, and we are aware of only one study demonstrating that nonpredictive peripheral cues lead to greater accuracy on valid than on invalid trials (Henderson \& Macquistan, 1993). This one study used multiple masks, making it impossible to determine whether the observed validity effects resulted from an allocation of resources to the cued location or a modulation of decision processes. Thus, the nature of the attention effects produced by nonpredictive peripheral cues is currently an open question.

It seems natural to suppose that an automatic attentional control mechanism would influence early, perceptual, processes rather than later, decision, processes. However, the fast time course of peripheral cuing suggests that the automatic capture of attention operates at least partly at the decision level. Specifically, reaction time studies have indicated that nonpredictive peripheral cues may yield large attention effects even when the cue-target stimulus onset asynchrony (SOA) is as brief as $0 \mathrm{msec}$ (Posner \& Cohen, 1984); it is likely that resource allocation requires a substantial period of time between the cue and the target (Luck et al., 1996), whereas decision effects could occur even if the cue followed the target display (as in the partial-report procedure developed by Sperling, 1960, to study iconic memory). It therefore is plausible to suppose that at least some of the attention effects produced by nonpredictive peripheral cues reflect uncertainty reduction at the level of a decision process that occurs after perceptual coding is complete. ${ }^{3}$

\section{EXPERIMENT 1}

Experiment 1 was an attempt to differentiate between the resource allocation model and the uncertainty reduction model in the context of nonpredictive peripheral cues by comparing a single-mask condition in which only the target location was masked and the location of the target was very obvious with a multiple-mask condition in which all four locations were masked and it was difficult to determine which location contained the target. Because there was no uncertainty about the location of the target in the single-mask condition, any attention effects observed in this condition can be attributed to resource allocation. Attention effects observed in the multiplemask condition may reflect a combination of resource allocation and uncertainty reduction, and as a first approximation we assume that the contribution of uncertainty reduction in the multiple-mask condition can be estimated by subtracting the attention effect observed in the single-mask condition from the attention effect observed in the multiple-mask condition. For example, if invalid cues lead to a $40 \%$ impairment relative to valid cues in the multiple-mask condition and a $10 \%$ impairment in the single-mask condition, we will assume that there is a $10 \%$ effect due to resource allocation in both conditions and an additional $30 \%$ effect due to uncertainty reduction in the multiple-mask condition. This approach assumes that the effects of perceptual-level resource allocation are not influenced by the presence of noise at the nontarget lo- 


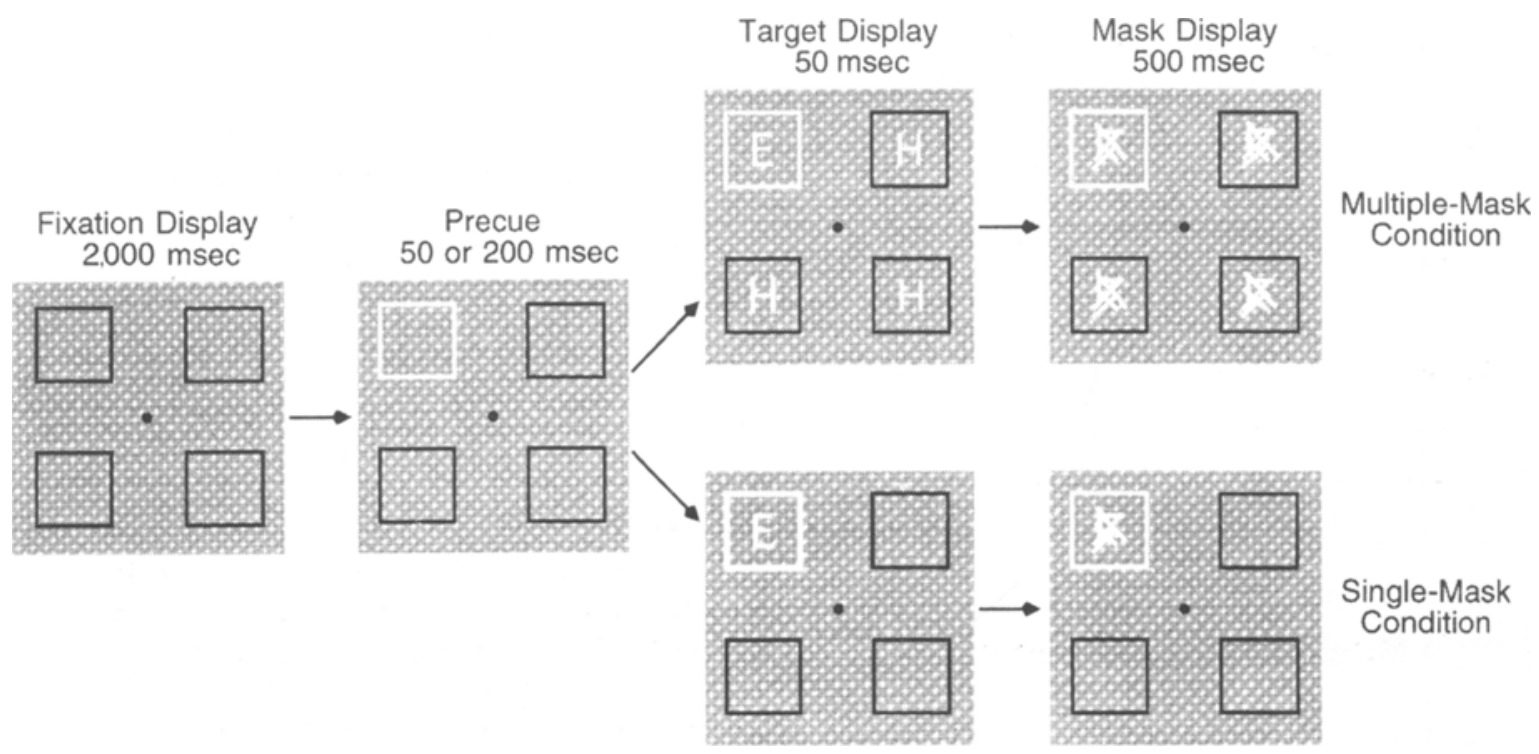

Figure 2. Example trial sequences for Experiment 1. Note that, for the purpose of visibility, the letters and masks are drawn much larger here than they appeared in the experiment; in reality, the location marker boxes were approximately five times as large as the letters. This sequence represents a valid trial, in which the cue (the white box) was at the same location as the target (the letter $\mathbf{E}$ ). In the multiple-mask condition, the foil letter $\mathrm{H}$ appeared at the three locations not occupied by the target, and all four locations were subsequently masked. In the single-mask condition, no foils were presented and only the target location was masked.

cations, which is likely to be true under the conditions of the present study, in which the targets were relatively simple and the locations widely spaced. There may be conditions under which this assumption is false, however, such as when the locations are close together and illusory conjunctions are likely to occur in the absence of attention (see Cohen \& Ivry, 1989, 1991).

In most previous comparisons of resource allocation and uncertainty reduction, target location uncertainty was created by presenting a target at one location and masks at all locations (e.g., Luck et al., 1996; Shiu \& Pashler, 1994). Although the presence of multiple masks may make target localization somewhat difficult, this procedure only partially obscures the location of the target. In the present study, we increased the difficulty of target localization even further by presenting nontarget foil stimuli simultaneously with the target stimulus in the multiplemask condition (Figure 2). Thus, the multiple-mask condition differed from the single-mask condition in terms of the presence of foils as well as the presence of multiple masks, but both of these differences were designed to achieve the same goal, namely target location uncertainty.

In addition to varying the presence or absence of location uncertainty, we also varied the cue-target SOA in this experiment to assess the time course of the attention effects.

\section{Method}

Subjects. The subjects consisted of 12 University of lowa students who received a combination of course credit and monetary compensation. All subjects were between the ages of 18 and 30 and reported normal or corrected-to-normal visual acuity.
Stimuli. The stimuli used in this experiment are illustrated in Figure 2. A fixation point and four location markers were continuously visible on a gray background $\left(2.6 \mathrm{~cd} / \mathrm{m}^{2}\right)$ throughout each trial block. Each location marker consisted of a $2.0^{\circ} \times 2.0^{\circ}$ black outline of a square, centered $4.2^{\circ}$ from fixation at equal distances $\left(3.0^{\circ}\right)$ from the vertical and horizontal meridians. A location was cued by changing the location marker at that location from black $\left(<0.001 \mathrm{~cd} / \mathrm{m}^{2}\right)$ to white $\left(22.8 \mathrm{~cd} / \mathrm{m}^{2}\right)$. The target stimulus was centered within one of the four boxes and was a white letter $\left(22.8 \mathrm{~cd} / \mathrm{m}^{2} ; 0.3^{\circ}\right.$ wide and $0.4^{\circ}$ high), selected at random on each trial from the set of E, F, I, or $\mathrm{T}$. In the multiple-mask condition, the remaining three boxes contained the letter $\mathrm{H}$, which served as a foil. In the single-mask condition, the target letter appeared without any simultaneous foil stimuli.

The target display was followed by a mask display, which consisted of a set of randomly oriented lines covering the area of each letter. In the single-mask condition, only the location of the target letter was masked. In the multiple-mask condition, all four locations were masked.

Procedure. Each trial began with a 2,000-msec fixation display, during which only the fixation point and location-marker boxes were present. This was followed by a brightening of the location marker at one of the four locations, which served as the cue. After an SOA of either 50 or $200 \mathrm{msec}$, the target display was presented for a duration of $50 \mathrm{msec}$. The target display was then replaced by the mask display, which was visible for $500 \mathrm{msec}$. The cue box was changed from white back to black at the offset of the mask display. After a 2,000-msec delay, feedback was provided to the subject. The feedback consisted of the target letter presented at the location of the fixation point for $500 \mathrm{msec}$. The subject was required to press one of four buttons to indicate the identity of the target, and this response had to occur before the onset of the feedback display. The next trial began immediately after the offset of the feedback display.

The target stimulus appeared at the cued location on $25 \%$ of trials and at each of the three uncued locations on $25 \%$ of trials. Thus, 

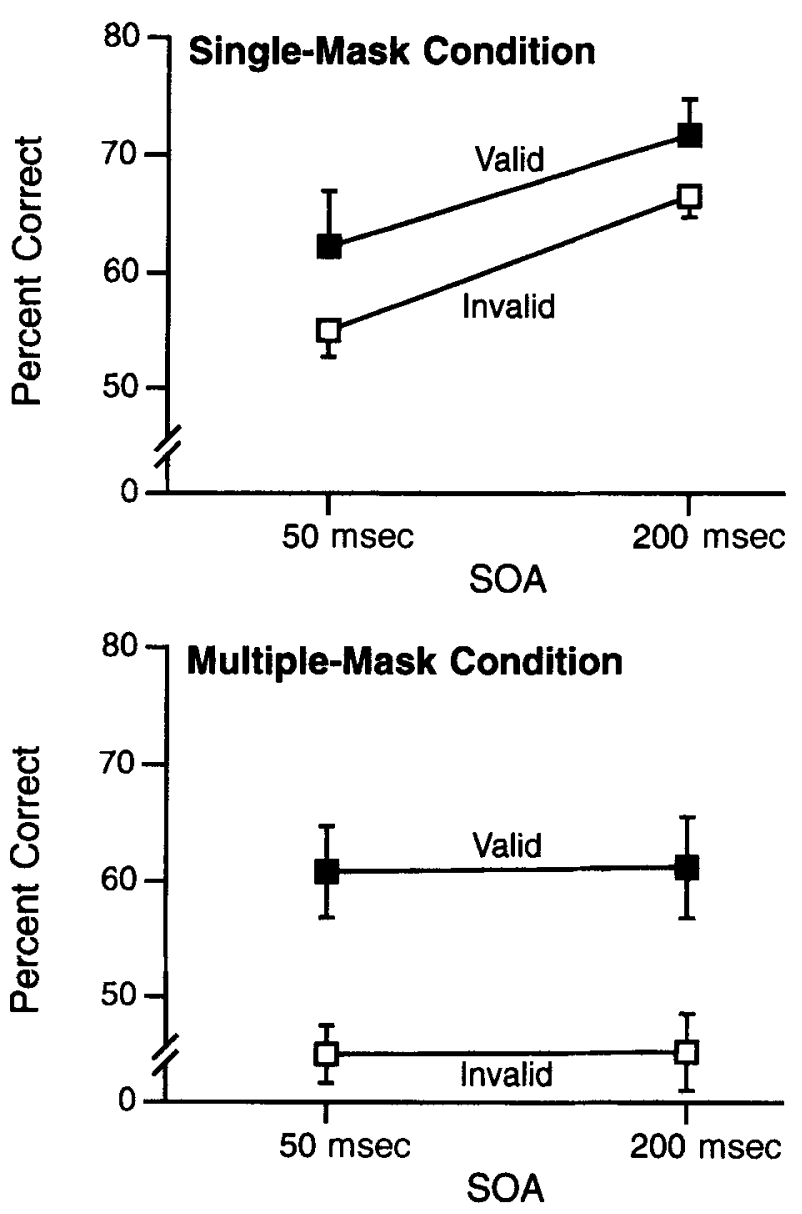

Figure 3. Mean accuracy (percent correct) as a function of masking condition and stimulus onset asynchrony (SOA) for Experiment 1. Error bars indicate \pm 1 SEM.

the cue provided no information about the locatton of the target. However, for purposes of comparison with studies using predictive cues, we use the term valid to describe trials on which the target appeared at the cued location and the term invalid to describe trials on which the target appeared at an uncued location. The main experimental manipulations in this experiment were therefore cue validity (valid or invalid), masking condition (single mask or multiple mask), and cue-target SOA ( 50 or $200 \mathrm{msec}$ ).

The single-mask and multiple-mask conditions were run in separate trial blocks, with 64 trials per block and five blocks for each condition. Each subject thus received 40 valid and 120 invalid trials at each SOA in each condition. Single-mask and multiple-mask blocks were alternated; half of the subjects received a single-mask block first and the others received a multiple-mask block first. Each subject served in a single session consisting of a series of practice blocks followed by the experimental blocks. The practice blocks were used to adjust the contrast between the letter stimuli and the masks so that each subject's performance on valid trials in the singlemask condition yielded an accuracy of $70 \%-80 \%$ correct. The contrast was also adjusted during the experimental blocks if accuracy in the single-mask condition began to deviate substantially from the $70 \%-80 \%$ level. Adjustments were made only after a pair of singlemask and multiple-mask trial blocks, however, so that mean contrast was always matched across conditions. These adjustments were necessary for only a small number of subjects.
Eye movements were monitored with an ISCAN RK-410 pupiltracking system. Off-line analysis of the eye movement data was used to elıminate trials on which eye movements occurred during the time between the onset of the cue and offset of the target. Fewer than $15 \%$ of trials were rejected in this manner for each subject, and these trials were excluded from all analyses. Across-trial averages of the eye movement records were also computed for each subject, and these averages were used to eliminate subjects whose averages showed eye movements in excess of $0.25^{\circ}$ in the direction of the cued location; 1 subject was replaced for this reason.

\section{Results}

Figure 3 shows accuracy as a function of cue validity for the single-mask and multiple-mask conditions. In this figure and all analyses reported below, the data were collapsed across the four possible target positions. In the single-mask condition, accuracy was approximately $6 \%$ greater on valid trials than on invalid trials, and accuracy generally increased with increasing SOA. In the multiplemask condition, valid-trial accuracy was approximately $15 \%$ greater than invalid-trial accuracy, but there was little change as a function of SOA. Thus, cue validity effects were observed in both the single-mask and multiplemask conditions, but the magnitude of the effect was more than twice as large in the multiple-mask condition as it was in the single-mask condition.

A three-factor repeated measures analysis of variance (ANOVA) was conducted on the accuracy data with factors of SOA ( 50 vs. $200 \mathrm{msec}$ ), validity (valid vs. invalid), and masking condition (single mask vs. multiple mask). This ANOVA revealed a significant main effect of masking condition $[F(1,11)=16.30, p<.01]$, due to the overall greater accuracy observed in the single-mask condition. There was also a significant validity main effect $[F(1,11)=18.18, p<.01]$, reflecting the greater accuracy on valid trials than on invalid trials. However, there was also a significant interaction between masking condition and validity $[F(1,11)=7.83, p<.05]$, due to the larger size of the validity effect in the multiple-mask condition. A significant main effect of SOA was also obtained $[F(1,11)=24.14, p<.01]$, due to overall greater accuracy at the $200-\mathrm{msec}$ SOA. The SOA effect was primarily present in the single-mask condition, which led to a significant interaction between masking condition and $\operatorname{SOA}[F(1,11)=13.42, p<.01]$. The three-way interaction was not significant, however. These analyses confirm the main observations of this experiment - namely, that accuracy was generally greater on valid than on invalid trials and that the validity effect was larger in the multiplemask condition than in the single-mask condition.

To determine whether a significant validity effect was present in each masking condition, planned comparisons were conducted in which the data from each condition were analyzed in separate ANOVAs. In the single-mask condition, this analysis yielded a significant main effect of validity $[F(1,11)=5.92, p<.05]$, along with a significant main effect of SOA $[F(1,11)=38.30, p<.001]$. In the multiple-mask condition, only the main effect of validity was significant $[F(1,11)=18.22, p<.01]$. Thus, signif- 
icant validity effects were observed for both the singlemask and multiple-mask conditions, although this effect was significantly larger in the multiple-mask condition.

\section{Discussion}

The presence of a significant cue validity effect in the single-mask condition of this experiment indicates that nonpredictive peripheral cues may attract processing resources, just as in the case of predictive cues (Cheal \& Gregory, 1997; Henderson, 1996; Luck et al., 1996). In addition, the finding of substantially larger effects in the multiple-mask condition than in the single-mask condition suggests that peripheral cues also automatically influence decision processes, biasing the subjects to report information presented at the cued location even though this information is no more likely to reflect the target than information at the uncued locations. This second conclusion is somewhat less certain than the first conclusion, however, because it is based on the assumption that the effects of resource allocation are the same in the singlemask and multiple-mask conditions. However, even if this assumption is violated to some extent, it seems unlikely that resource allocation differences between the singlemask and multiple-mask conditions could explain the very large difference in the size of the attention effects across these conditions. Thus, as in the case of predictive cues, nonpredictive peripheral cues appear to influence both resource allocation and decision processes.

In the single-mask condition, accuracy was somewhat greater at the longer cue target SOA than at the shorter cue-target SOA, but there was no such effect in the multiple-mask condition. We have no explanation for this difference. However, SOA did not interact with cue validity in either condition, nor was there a significant threeway interaction between SOA, masking condition, and cue validity, so this effect does not appear to reflect differences in the time course of cue-directed attention.

On the basis of previous reaction time experiments with nonpredictive peripheral cues, one might expect to observe larger cue validity effects at the 50 -msec SOA than at the 200-msec SOA. There are two likely explanations for the absence of this pattern in the present study. First, although some attention-related modulations of processing may have similar effects on both reaction time and accuracy, other attention effects may not. For example, faster reaction times on valid trials may be partially caused by a reduction in the amount of information that is accumulated before a response is triggered (i.e., a lower response threshold), but this sort of attentional mechanism would lead to lower accuracy on valid trials rather than greater accuracy. Thus, the finding of greater cue validity effects at short SOAs in reaction time experiments but not in accuracy experiments may be a result of the presence of multiple attentional mechanisms that operate at different stages (Handy, Kingstone, \& Mangun, in press; Luck, 1995).

A second possible explanation for the lack of a larger cue validity effect at the shorter SOA is that this experi- ment was designed to minimize inhibition of return, which may be responsible for the decline in cue validity effects typically observed at longer SOAs when uninformative cues are used. Specifically, previous research has shown that inhibition of return is initiated by the offset of the cue rather than its onset (Tassinari, Aglioti, Chelazzi, Peru, \& Berlucchi, 1994), and the cues in the present experiment did not offset until $500 \mathrm{msec}$ after target offset. Thus, the constant size of the cue validity effect at the 50and 200-msec SOAs may simply refiect a lack of inhibition of return.

\section{EXPERIMENTS 2 AND 3}

Although the cues in Experiment 1 provided no information about the location of the target, the observed vaiidity effects may not have been completely automatic, because nothing about the experimental design discouraged the subjects from orienting attention to the cues. Folk and colleagues have argued that the automatic capture of attention by a stimulus depends on the observer's "attentional control settings," which may be biased toward or away from a given stimulus type (Folk, Remington, \& Johnston, 1992, 1993; Yantis, 1993). When observers search for color-defined targets, for example, luminance transients no longer automatically capture attention. Folk et al. $(1992,1993)$ also proposed that the human visual system may have default settings that are sensitive to stimuli such as the luminance transients used as cues in Experiment 1 , which would explain why such cues capture attention even when luminance increments are not a targetdefining feature. Further, although they proposed that the capture of attention is contingent upon task demands, they also posited that once an observer's attentional control settings have been configured for a given task, stimuli that match the control settings will cause an involuntary orienting of attention. This proposal is therefore called the contingent involuntary orienting hypothesis.

Folk et al. (1992) tested this hypothesis by factorially combining color-defined and onset-defined nonpredictive cues with color-defined and onset-defined targets, predicting that cue validity effects would be observed only when the cue and target were defined by the same stimulus dimension. Onset cues consisted of the appearance of four dots around one of the possible target locations, and color cues consisted of the simultaneous appearance of four red dots around one of the possible target locations and four white dots around the remaining locations. The onset target consisted of a single $\mathrm{X}$ or an $=$ character, and the color target consisted of a red $\mathrm{X}$ or an $=$ character accompanied by three white $\mathrm{X}$ or $=$ characters that served as nontarget foils. Reaction times were measured for the discrimination between the $\mathrm{X}$ and $=$ target alternatives, and the observers were found to be faster on valid trials than on invalid trials, but only for the onset-cue/onsettarget and color-cue/color-target conditions (see especially Experiment 3 of Folk et al., 1992). No significant cuing effects were found with a luminance cue combined 
with a color-defined target or with a color-defined cue combined with a luminance-defined target, consistent with the hypothesis that the observer's task-defined attentional set influences the capture of attention by a nonpredictive cue stimulus.

It is important to note that there was no uncertainty about the location of the target for either onset-defined or color-defined targets in the Folk et al. (1992) study; the onset-defined targets were the only target-like stimuli in the display, and the color-defined targets could be localized very easily by their distinctive color. This design therefore provided no opportunity to observe decisionlevel attention effects, leaving open the possibility that decision-level attention effects might be fully automatic, operating even when the cue is defined by a luminance change and the observers are searching for color-defined targets. In addition, Folk et al. (1992) focused on reaction time, and it is possible that an entirely different pattern of results would have been observed if the experimental design had focused on accuracy. The purpose of Experiment 2 was to evaluate these possibilities and to more fully assess the automaticity of the attentional capture observed in Experiment 1.

In Experiment 2, the subjects were encouraged to adopt a bias toward color, thus overriding any default biases toward the luminance increments that defined the cues. However, it was not possible simply to use a target of one color and foil stimuli of another color, as in the study of Folk et al. (1992), because this eliminates uncertainty about which stimulus is the target. Instead, we used a mixture of trial types, some of which had a distinctively colored target and others of which did not. For example, in some trial blocks the subject was told that the target would be a red item; on $50 \%$ of trials, the target was red and the foils were green (singleton trials), and on the remaining trials the target and the foils were all red (homogeneous trials). Because the singleton and homogeneous trials were randomly intermixed, the subjects were expected to maintain an attentional bias toward uniquely colored items, thus overriding any default biases that might cause attention to be captured by the luminance-defined cues. This manipulation also made it possible to assess the effects of attention both with and without location uncertainty. On singleton trials, the target's distinctive color made its location very obvious, just as in the single-mask condition of Experiment 1. On homogeneous trials, however, the location of the target was not obvious, just as in the multiple-mask condition of Experiment 1. Thus, cue validity effects on singleton trials may be attributed to resource allocation, whereas cue validity effects on homogeneous trials may reflect a combination of resource allocation and location uncertainty reduction.

The target was presented in a singleton color on only $50 \%$ of trials in Experiment 2, and it is possible that this was not sufficient to engender a strong bias toward color singletons and to override any default biases toward lu- minance onsets. Experiment 3 was conducted to examine this possibility. Experiment 3 was exactly the same as Experiment 2 , except that the singleton and homogeneous trials were presented in separate blocks rather than being mixed. In the singleton blocks, therefore, the target was always a color singleton, which should have led to a strong bias to attend to the color singleton and to ignore luminance increments, just as in the study of Folk et al. (1992). If the use of a mixture of singleton trials and homogeneous trials in Experiment 2 did not engender a strong bias toward the color singletons, one would expect a stronger effect of the luminance cues in that experiment than in Experiment 3. If, however, the luminance cue produces equivalent effects in Experiments 2 and 3, then it would be difficult to argue that the cuing effect in Experiment 2 was due to the fact that only $50 \%$ of the targets in that experiment were color singletons.

An additional methodological change was also made in these experiments. Yantis and his colleagues have provided evidence indicating that it is the appearance of a new object rather than an increment in luminance that is the cause of automatic attentional capture (Hillstrom \& Yantis, 1994; Yantis \& Hillstrom, 1994), but the cue used in Experiment 1 consisted of a brightening of a preexisting location marker rather than the appearance of a new object. In Experiments 2 and 3, the cue appeared as a new object rather than as an increment in the luminance of a preexisting object, thus optimizing the automatic capture of attention.

\section{Method}

Subjects. The subjects consisted of 24 University of lowa students, 12 in Experiment 2 and 12 in Experiment 3 . All subjects were between the ages of 18 and 30 , reported normal or corrected-tonormal visual acuity, and were paid to participate.

Stimuli and Procedure. The stimuli and procedure for Exper1ments 2 and 3 were the same as those used in the multiple-mask condition of Experiment 1, with the following exceptions. The target was red $\left(u^{\prime}=.444, v^{\prime}=.526\right)$ for half of the trial blocks and green $\left(u^{\prime}=.137, v^{\prime}=.556\right)$ for the remaining blocks (the subjects were informed of the target color prior to each block). On homogeneous trials, the foil items were drawn in the same color as the target item; on singleton trials, the foil items were drawn in the color that was not used for the target. The mask for a given letter was drawn in the same color as that letter. The location markers in these experiments were reduced in size to $1.3^{\circ} \times 1.3^{\circ}$, but the cue remaned unchanged at $2.0^{\circ} \times 2.0^{\circ}$. Thus, the cue was a white outline square drawn on the gray background of the screen, surrounding one of the black location markers. This is illustrated in Figure 4.

Each trial block consisted of 64 trials, and each subject received 12 blocks, half with red as target and half with green as target. Redtarget and green-target blocks were alternated, and the startıng color was counterbalanced across subjects. In Experiment 2, singleton and homogeneous trials were randomly intermixed within each trial block. In Experiment 3, one half of each block consisted entirely of singleton trials and the other half consisted entırely of homogeneous trials; singleton trials came first for half of the subjects and homogeneous trials came first for the other half. The subjects were informed of the type of trials that would be presented at the beginning of each half-block. 


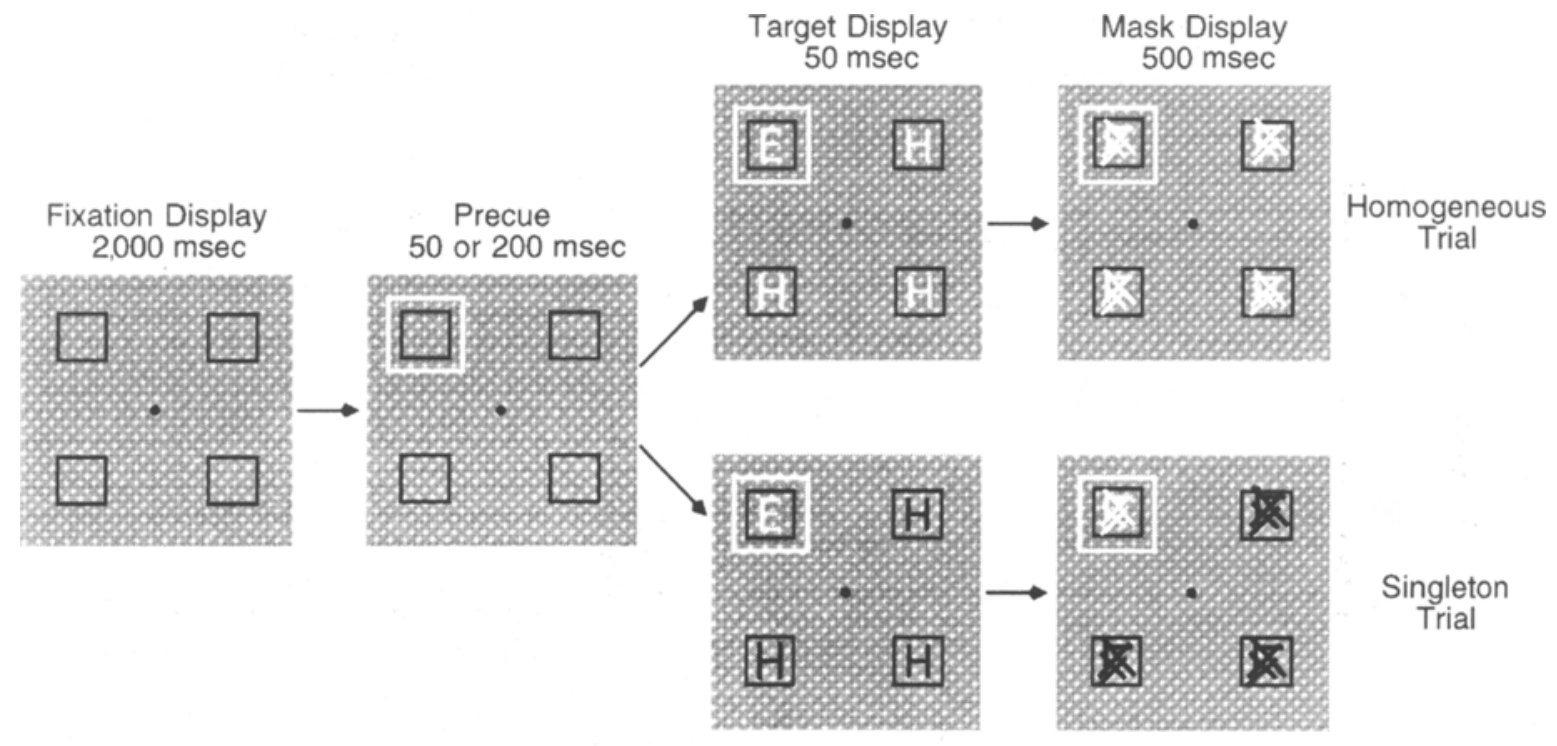

Figure 4. Example trial sequences for Experiment 2. As in Figure 2, the digits are drawn larger here than they actually appeared in the experiment. The letters and masks were drawn in red and green rather than in the black and white shown here.

Each session began with a series of practice blocks in which the brightness of the masks was adjusted so that each subject's overall accuracy was approximately $80 \%$ correct. Brightness was adjusted independently for the red and green masks. Mask brightness was also adjusted during the experimental blocks if accuracy began to deviate substantıally from the $80 \%$ level. Adjustments were made only after a pair of red-target and green-target blocks, however, so that mean mask brightness was always matched across conditions. These adjustments were necessary only for a small number of subjects.

Eye movements were monitored by means of electrooculogram (EOG) recordings. The EOG was recorded as a bipolar signal from electrodes located adjacent to the external canthi for monitoring horizontal eye movements and from electrodes above and below the left eye to monitor vertical eye movements. Off-line analysis of the eye movement data was used to elıminate trials on which eye movements occurred during the time between the onset of the cue and the offset of the target. Fewer than $15 \%$ of trials were rejected in this manner for each subject, and these trials were excluded from all analyses. Across-trial averages of the eye movement records were also computed for each subject, and these averages were used to eliminate subjects whose averages showed eye movements in excess of $0.25^{\circ}$ in the direction of the cued location; 2 subjects were replaced for this reason in Experiment 2, and 3 were replaced in Experıment 3.

\section{Results}

Figure 5 shows accuracy as a function of cue validity for singleton and homogeneous trials in Experiments 2 and 3 . The cue validity effects were virtually identical across Experiments 2 and 3, and they were also quite similar to those found in Experiment 1. For both singleton and homogeneous trials, accuracy was greater on valid trials than on invalid trials, independent of SOA. In addition, the validity effect was approximately twice as large for homogeneous trials as for singleton trials.

Experiment 2 analyses. The accuracy data from Experiment 2 were analyzed with a three-factor repeated measures ANOVA, with factors of SOA ( $50 \mathrm{vs.} 200 \mathrm{msec}$ ), validity (valid vs. invalid), and trial type (singleton vs. homogeneous). The main effect of validity was highly significant $[F(1,11)=29.16, p<.001]$, and the larger size of the validity effect on homogeneous trials led to a significant interaction between trial type and validity $[F(1,11)=$ $8.20, p<.02]$. Accuracy was also generally higher for singleton trials than for homogeneous trials, leading to a significant main effect of trial type $[F(1,11)=21.93, p<$ $.001]$. In addition, accuracy tended to be greater at the 200-msec SOA than at the 50-msec SOA, leading to a significant main effect of SOA $[F(1,11)=16.58, p<.002]$, but this effect did not interact significantly with trial type or cue validity.

To assess the effects of attention for singleton and homogeneous trials separately, planned comparisons were performed in which the data for each trial type were analyzed in separate ANOVAs with factors of validity and SOA. For both singleton and homogeneous trials, the greater overall accuracy on valid trials than on invalid trials led to a significant main effect of validity $[F(1,11)=$ $13.36, p<.005$, and $F(1,11)=31.77, p<.001$, respectively]. The main effect of SOA was also significant for both singleton and homogeneous trials $[F(1,11)=22.75$, $p<.001$, and $F(1,11)=5.07, p<.05$, respectively $]$. SOA and validity did not interact significantly for either trial type, however.

Experiment 3 analyses. The same analyses were also conducted for Experiment 3, and the results were nearly identical. The main effect of validity was highly significant $[F(1,11)=63.86, p<.001]$, and the larger size of the validity effect on homogeneous trials led to a significant interaction between trial type and validity $[F(1,11)=$ $7.00, p<.03$ ]. Accuracy was also generally higher for singleton trials than for homogeneous trials, leading to a significant main effect of trial type $[F(1,11)=15.34, p<$ 


\section{Experiment 2 Mixed}
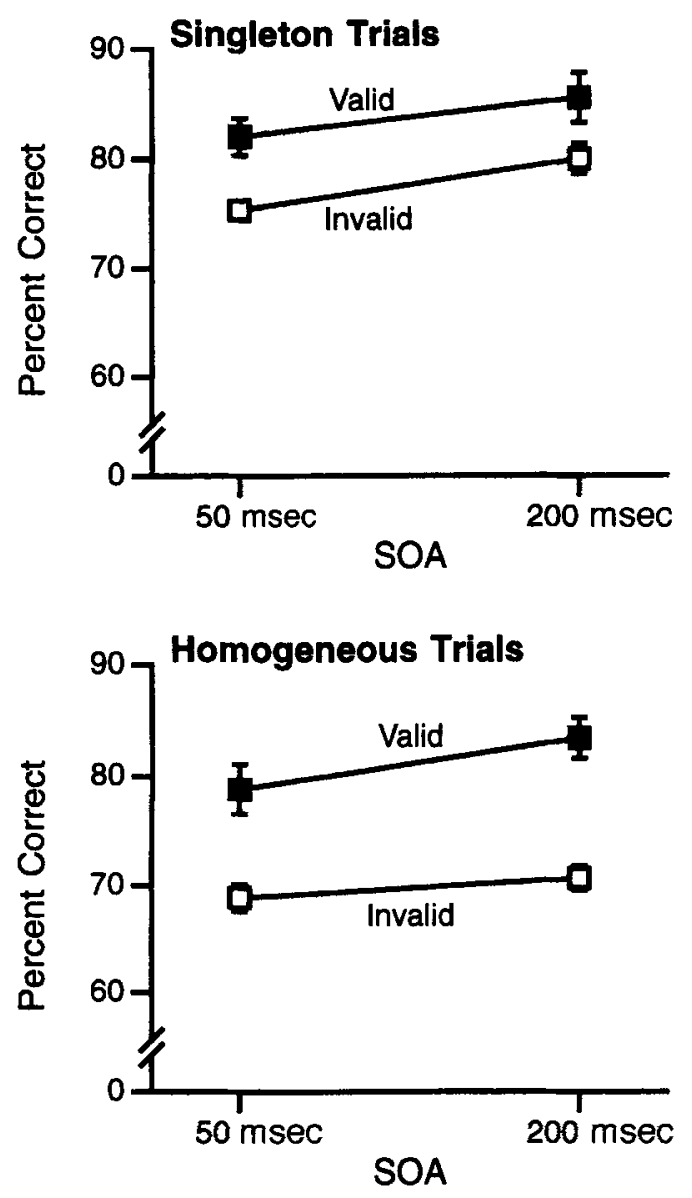

\section{Experiment 3 Blocked}
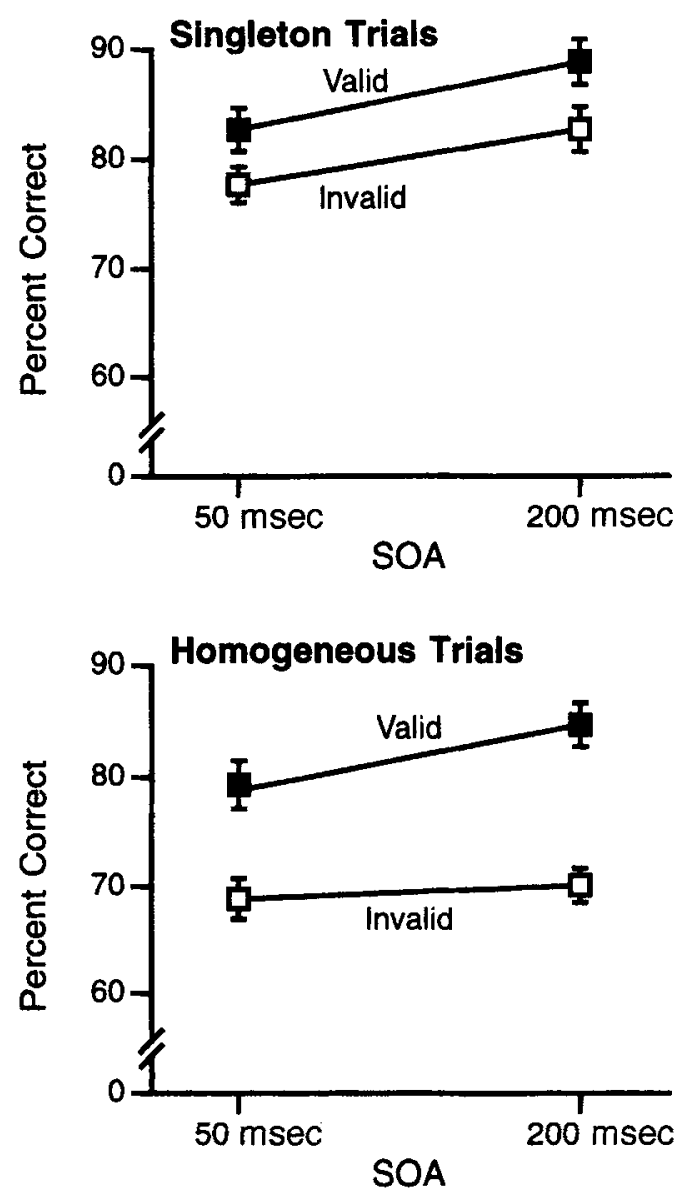

Figure 5. Mean accuracy (percent correct) as a function of trial type and stimulus onset asynchrony (SOA) for Experiments 2 and 3. Error bars indicate \pm 1 SEM.

$.005]$. In addition, accuracy tended to be greater at the 200 -msec SOA than at the $50-\mathrm{msec}$ SOA, leading to a significant main effect of SOA $[F(1,11)=20.11, p<$ $.001]$, but this effect did not interact significantly with trial type or cue validity.

In a set of planned comparisons, the greater overall accuracy on valid than on invalid trials led to a significant main effect of validity for both the singleton trials $[F(1,11)=24.50, p<.001]$ and the homogeneous trials $[F(1,11)=32.92, p<.001]$. The greater accuracy at the 200- relative to the 50-msec SOA led to a significant main effect of SOA for both singleton and homogeneous trials $[F(1,11)=14.71, p<.005$, and $F(1,11)=8.05, p<.02$, respectively]. SOA and validity did not interact significantly for either trial type, however.

Comparison of Experiments 2 and 3. To determine whether blocking the singleton and homogeneous trials in Experiment 3 yielded different results from those obtained in Experiment 2, the data from both experiments were entered into a single mixed-design ANOVA, with a between-subjects factor of experiment and within-subjects factors of SOA, validity, and trial type. Neither the main effect of experiment nor any of the interactions involving experiment approached significance ( $p>.30$ in all cases). Thus, it is very unlikely that the subjects in Experiment 2 failed to be biased toward the color singletons due to the fact that only half of the targets were singletons, because this was not a possibility in Experiment 3 and yet the results were statistically indistinguishable.

\section{Discussion}

The finding of significant validity effects in Experiment 2 indicates that nonpredictive cues can capture attention even when the cue is defined by a luminance increment and the subjects are encouraged to be biased toward a distinctive color. This suggests that the attentional capture produced by a luminance increment has a relatively high level of automaticity. In addition, the finding 
of a reliable validity effect for the singleton trials and an even larger validity effect for the homogeneous trials suggests that the automatic capture of attention influences both resource allocation and decision processes. That is, the validity effects observed on singleton trials probably reflect the allocation of resources to the cued location, and the larger validity effects observed on homogeneous trials probably reflect both the allocation of resources and an effect of uncertainty reduction. Thus, Experiments 1,2, and 3 all indicate that the automatic capture of attention leads to changes in both resource allocation and decision processes.

These findings appear to conflict with the results reported by Folk et al. (1992), who found no validity effects when the cue was defined by a luminance onset and the target was defined by its distinctive color (comparable to the singleton trials of the present experiment). There are several possible explanations for this discrepancy. First, the Folk et al. (1992) study examined reaction time for unmasked stimuli rather than accuracy for masked stimuli, and these measures may be differentially influenced by attention (possibly due to differences in strategy; see Handy et al., in press). Moreover, this may have been exacerbated by the fact that Folk et al. (1992) used conditions that were analogous to the singleton trials of the present experiment, which yielded significantly smaller validity effects than the homogeneous trials.

It is also possible that the use of singleton targets in Experiment 2 failed to bias the subjects toward color singletons and away from luminance onsets. However, there are several reasons why this is unlikely. First, the fact that the target was a singleton on only half of the trials in Experiment 2 cannot easily explain a lack of bias toward the color singletons, because the effects were no different in Experiment 3 , in which all of the targets were singletons over a period of many trials. Second, the subjects were clearly using the information provided by the distinctive color of the target on singleton trials, because performance was significantly better on singleton trials than on homogeneous trials. Third, the stimuli and procedures used in these experiments were quite similar to those used by Folk et al. (1992), except that the targets were masked and accuracy was stressed rather than speed. Thus, the singleton trials in Experiments 2 and 3 should have led to the same level of bias toward singletons as in the study of Folk et al. (1992). Of course, it is always possible that some yet-to-be-specified changes in procedure could yield a stronger singleton bias and therefore a smaller effect of the luminance onset cues, but the present results indicate that under at least some conditions, luminance onsets will capture attention even when the stimuli and task are designed to bias subjects toward color singletons and away from luminance onsets.

\section{GENERAL DISCUSSION}

From these experiments, three main conclusions can be drawn about the nature of the attention effects produced by nonpredictive peripheral cues. First, the automatic attention effects produced by these cues can influence accuracy and not just reaction time. Surprisingly, we have been able to find only one previous study showing a significant improvement in accuracy on valid trials relative to invalid trials with nonpredictive peripheral cues (Henderson \& Macquistan, 1993), even though it is theoretically important to know whether nonpredictive cues influence accuracy as well as reaction time.

A second conclusion that can be drawn from this study is that nonpredictive peripheral cues may influence the allocation of limited perceptual processing resources, resulting in improved perceptual quality for stimuli presented at the cued location. This conclusion is based on the finding of greater accuracy on valid trials than on invalid trials in the single-mask condition of Experiment 1 and on the singleton trials in Experiments 2 and 3. According to the logic developed by Shiu and Pashler (1994), precues should have no effect on decision-level processes under such conditions, and these results can therefore be attributed to resource allocation. Moreover, these cuing effects appear to be highly automatic, because they were obtained even on the singleton trials of Experiment 3, in which subjects were presumably highly biased toward color singletons rather than luminance increments.

The third conclusion that can be drawn from this study is that the automatic capture of attention produced by nonpredictive peripheral cues leads to a modulation of decision-level processes that are interposed between perceptual and motor processes. This conclusion is based on the finding of larger cue validity effects when the observers could not easily ascertain the location of the target than when the target's location was clearly marked (i.e., larger effects in the multiple-mask condition of Experiment 1 and the homogeneous trials of Experiments 2 and 3 than in the single-mask condition and singleton trials). It would be very difficult for a perceptual-level model of attention to account for this very large difference in the magnitude of the cuing effects. The only difference between the single-mask and multiple-mask conditions in Experiment 1 was the presence of irrelevant information at the nontarget locations in the multiple-mask condition, and given the wide spacing between locations, there is no reason to suspect that the presence of this irrelevant information would greatly alter the effects of perceptuallevel attentional mechanisms. Moreover, stimuli were present at the irrelevant locations on both the singleton and homogeneous trials in Experiments 2 and 3, making it even less likely that the larger cuing effect in the homogeneous condition was due to an interaction between attention and sensory factors. Furthermore, there is no reason to suspect that the presence of a singleton color should have caused a reduction in the perceptual-level effects of the nonpredictive luminance onset cues in Experiments 2 and 3. These results therefore indicate that nonpredictive peripheral cues automatically bias decision processes so that stimuli presented at cued locations are more likely to be reported than stimuli presented at uncued locations, 
even if the cued and uncued stimuli are represented with equivalent perceptual quality.

Throughout this article, we have contrasted the allocation of perceptual processing resources with decisionlevel attention effects, but it is important to note that decision-level effects can potentially be combined with changes in postperceptual resource allocation. In particular, the finding of larger cue validity effects for the multiple-mask condition and homogeneous trials than for the single-mask condition and singleton trials can be explained by a model in which attention operates to select which perceptual representations are transferred into a limited-capacity working memory system. According to this model, transferring information about a single stimulus into working memory requires several hundred milliseconds (see, e.g., Giesbrecht \& Di Lollo, 1998; Shapiro \& Raymond, 1994), and it is therefore impossible to transfer the perceptual information from all four locations into working memory without substantial decay. Performance on valid trials in the multiple-mask condition and homogeneous trials would therefore be greatly enhanced by always transferring the information from the cued location into working memory, although this would result in poor performance on invalid trials due to the decay of information that was presented at uncued locations. For the single-mask condition and singleton trials, however, it seems likely that only the information from the target location would be transferred into working memory, regardless of whether the target was validly or invalidly cued. Thus, the presence of capacity limitations in working memory may be partially responsible for the finding of larger attention effects in the multiple-mask condition than in the single-mask condition. This could still be viewed as a special case of the decision-level model, however, because the cue is assumed to influence a postperceptual decision about the stimuli (i.e., which stimuli to retain in working memory) rather than influencing the initial analysis of target identity. This model also provides an explanation for an apparent conundrum: If we assume that decision processes are controlled in a top-down manner, it seems odd that they would be influenced by bottomup nonpredictive cues. However, given that working memory is highly limited, the likely importance of luminance increments in the natural world makes it reasonable to suppose that such objects would have priority for transfer into working memory and for decisions that are based on the contents of working memory.

\section{REFERENCES}

Cheal, M., \& Gregory, M. (1997). Evidence of limited capacity and noise reduction with single-element displays in the location-cuing paradigm. Journal of Experimental Psychology. Human Perception \& Performance, 23, 51-71.

Cheal, M. L., \& Lyon, D. R. (1991). Central and peripheral precuing of forced-choice discrimination. Quarterly Journal of Experimental Psychology, 43A, 859-880.

Cohen, A., \& Ivry, R. B. (1989). Illusory conjunctions inside and outside the focus of attention. Journal of Experimental Psychology Human Perception \& Performance, 15, 650-663.
COHEN, A., \& IVry, R. B. (1991). Density effects in conjunction search: Evidence for a coarse location mechanısm of feature integration. Journal of Experimental Psychology Human Perception \& Performance, 17, 891-901.

Egeth, H. E., \& Yantis, S. (1997). Visual attention: Control, representation, and time course. Annual Review of Psychology, 48, 269297.

Eriksen, C. W., \& Hoffman, J E. (1974). Selective attention: Noise suppression or signal enhancement? Bulletin of the Psychonomic Society, 4, 587-589

Fol. , C. L., Remington, R. W., \& Johnston, J. C. (1992). Involuntary covert orienting is contingent on attentional control settings. Journal of Experimental Psychology Human Perception \& Performance, 18. 1030-1044

Folk, C. L., Remington, R. W., \& Johnston, J. C. (1993). Contingent attentional capture: A reply to Yantıs (1993). Journal of Experimental Psychology Human Perception \& Performance, 19, 682-685.

GiesbreChT, B. L., \& Di Lollo, V. (1998). Beyond the attentional blink: Visual masking by object substitution. Journal of Experimental Psychology. Human Perception \& Performance, 24, 1454-1466.

Handy, T. C., Kingstone, A., \& Mangun, G. R. (in press). Spatial distribution of visual attention: Perceptual sensitivity and response latency. Perception \& Psychophysics.

Hawkins, H. L., Hil.tyard, S. A., Luck, S. J., Mouloua, M., DownING, C. J., \& Woodward, D. P. (1990). Visual attention modulates signal detectability. Journal of Experimental Psvchology Human Perception \& Performance, 16, 802-811.

Hawkins, H. L., Shafto, M. G., \& Richardson, K. (1988). Effects of target luminance and cue validity on the latency of visual detection. Perception \& Psychophusics, 44, 484-492

Henderson, J. M. (1996). Spatial precues affect target discrimınation in the absence of visual noise. Journal of Experimental Psvchology Human Perception \& Performance, 22, 780-787

Henderson, J. M., \& Macquistan, A. D. (1993). The spatıal distribution of attention following an exogenous cue. Perception \& Psvchophysics, 53, 221-230.

Hillstrom, A. P., \& Yantis, S. (1994). Visual motion and attentıonal capture. Perception \& Psychophysic's, 55, 399-411.

JONIDES. J. (1981). Voluntary versus automatic control over the mind's eye's movement. In J. [B.] Long \& A. [D.] Baddeley (Eds.), Attention and performance $I X$ (pp. 187-203). Hillsdale, NJ: Erlbaum.

Kahneman, D. (1973). Attention and effort. Englewood Cliffs, NJ. Prentice-Hall.

LAPPIN, J. S., \& UTTAL, W. R. (1976). Does prior knowledge facilitate the detection of visual targets in random noise? Perception \& Psychophysics, 20, 367-374.

Luck, S. J. (1995). Multiple mechanisms of visual-spatıal attentıon: Recent evidence from human electrophysiology. Behavioural Brain Research, 71, 113-123.

Luck, S. J., Hillyard, S. A., Mouloua, M., \& Hawkins, H. L. (1996). Mechanisms of visual-spatial attention: Resource allocation or uncertainty reduction? Journal of Experimental Psvchology Human Perception \& Performance, 22, 725-737.

Luck, S. J., Hillyard, S. A., Mouloua, M., Woldorff, M. G., Cl.ark, V. P., \& HaWKINS, H. L. (1994). Effects of spatial cuing on lumınance detectabılity: Psychophysical and electrophysıological evidence for early selection. Journal of Experimental Psychology. Human Perception \& Performance, 20, 887-904.

Palmer, J., Ames, C. T., \& Lindsey, D. T. (1993). Measuring the effect of attention on simple visual search. Journal of Experimental Psychology Human Perception \& Performance, 19, 108-130.

Posner, M. I., \& COHEN, Y. (1984). Components of visual orienting. In H. Bouma \& D. G. Bouwhuis (Eds.), Attention and performance $X$ Control of language processes (pp. 531-556). Hillsdale, NJ: Erlbaum

Reinitz, M. T. (1990). Effects of spatially directed attention on visual encoding. Perception \& Psychophysics, 47, 497-505

ShaPIRo, K. L., \& RAYmond, J. E. (1994). Temporal allocation of visual attention: Inhibition or interference. In D. Dagenbach \& T H. Carr (Eds.), Inhibitory processes in attention, memory, and language (pp. 151-188). San Diego: Academic Press.

SHAw, M. L. (1984). Division of attention among spatial locations: A 
fundamental difference between detection of letters and detection of luminance increments. In H. Bouma \& D. G. Bouwhuis (Eds.), Attention and performance $X$ (pp. 109-121). Hillsdale, NJ: Erlbaum.

ShIU, L., \& PASHler, H. (1994). Negligible effect of spatial precuing on identıfication of single digits. Journal of Experimental Psychology: Human Perception \& Performance, 20, 1037-1054.

SPERLING, G. (1960). The information available in bref visual presentatıons. Psychological Monographs, 74.

SPERLING, G., \& Dosher, B. A. (1986). Strategy and optimization in human information processing. In K. R. Boff, L. Kaufman, \& J. P. Thomas (Eds.), Handbook of perception and human performance (pp. 2-65). New York: Wiley.

Tassinari, G., Aglioti, S., Chelazzi, L., Peru, A., \& Berlucchi, G. (1994). Do peripheral non-1nformative cues induce early facilitation of target detection? Vision Research, 34, 179-189.

YANTIS, S. (1993). Stımulus-driven attentional capture and attentional control settings. Journal of Experimental Psychology: Human Perception \& Performance, 19, 676-681.

Yantis, S., \& Hillstrom, A. P. (1994). Stimulus-driven attentional capture Evidence from equiluminant visual objects. Journal of Experimental Psychology: Human Perception \& Performance, 20, 95107.

YANTIS, S., \& JONIDES, J. (1990). Abrupt visual onsets and selective attention: Voluntary versus automatic allocation. Journal of Experimental Psychology: Human Perception \& Performance, 16, 121-134.

\section{NOTES}

1. More precisely, the decision process is weighted in a continuous manner so that information presented at the cued location is given greater weight than information presented at uncued locations. Thus, an observer may occasionally report the percept arising from an uncued location if that percept is exceptionally strong.

2 . For the sake of simplicity, this article primarily discusses cuing studies that examined accuracy rather than reaction time as the primary dependent variable. Accuracy is simpler for two reasons. First, speedaccuracy tradeoffs frequently complicate the interpretation of reaction time effects, but are generally irrelevant when accuracy is measured without time pressures. Second, reaction time effects may be caused by a wider variety of processing stages than accuracy effects (e.g., changes in motor preparation are unlikely to influence accuracy in the absence of time pressures)

3. As discussed by Shiu and Pashler (1994), attention may influence decision processes that occur during perception as well as postperceptual decision processes. However, most investigators assume that the decision stage follows perception (Palmer, Ames, \& Lindsey, 1993; Sperling \& Dosher, 1986), and we will also adopt this assumption (although it has little effect on our conclusions).

(Manuscript received March 4, 1997; revision accepted for publication August 6, 1998.) 